\title{
Classification of Zero Divisor Graphs of Commutative Rings of Degrees 11,12 and 13
}

\author{
Nazar H. Shuker \\ nazarh_2013@yahoo.com \\ Husam Q. Mohammad \\ College of Computer Sciences and Mathematics \\ University of Mosul, Iraq
}

Received on: 6/2/2012

Accepted on: 3/4/2013

\section{ABSTRACT}

In 2005 Wang investigated the zero divisor graphs of degrees 5,6,9 and 10. In 2012 Shuker and Mohammad investigated the zero divisor graphs of degrees 7 and 8 . In this paper, we consider zero divisor graphs of commutative rings of degrees 11,12 and 13 .

Key word: Zero-divisor, Ring, Zero-divisor graph.

$$
\text { أ.د. نزار حمدون شكر بيانات قواسم الصفر للحلقات الابدالية ذات الدرجات 11, } 12 \text { و } 13 \text { أ.م.د. حسام قاسم محد } 13 \text { الحاسبات والرياضيات، جامعة الموصل }
$$

في عام 2005 درس Wang بيانات قواسم الصفر للحلقات الابدالية من الدرجات 9,6,5 و10. في عام 2012 درسShuker and Mohammad بيانات قواسم الصفر للدرجتين 7 و8 .في هذا البحث درسنا بيانات قواسم

$$
\text { الصفر للحلقات الابدالية من الدرجات 12,11 و 13. } 13 \text { المفتاحية : قواسم الصغر , حلقة , بيان قواسم الصفر. }
$$

\section{Introduction}

The concept of zero divisor graph of a commutative ring was introduced by Beck in [3], he let all elements of the ring be vertices of a graph. In [1] Anderson and Livingston introduced and studied the zero divisor graph whose vertices are the nonzero zero divisors.

Throughout this paper, all rings are assumed to be commutative rings with identity, and $Z(R)$ be the set of zero divisors. We associate a simple graph $\Gamma(R)$ to a ring $R$ with vertices $Z(R)^{*}=Z(R)-\{0\}$, the set of all non-zero zero divisors of $R$. For all distinct $x, y \in Z(R)^{*}$, the vertices $x$ and $y$ are adjacent if and only if $x y=0$. In [1] Anderson and Livingston proved that for any commutative ring $\mathrm{R}, \Gamma(\mathrm{R})$ is connected.

In [6], Wang investigated the zero divisor graphs of degree 5, 6, 9 and 10. In [5], we consider the zero divisor graphs of degree 7 and 8 . In this paper, we extend these results to consider the zero divisor graphs of commutative rings of degrees 11,12 and 13.

\section{Rings with $|\mathrm{Z}(\mathbf{R}) *|=11$}

The main aim of this section is to find all possible zero divisor graphs of 11 vertices and rings correspond to them.

Recall that if $\mathrm{R}$ is a finite ring, then every element of $\mathrm{R}$ either unit or zero divisor [2]. In [6] Wang proved the following result.

Lemma 2.1: Let $\left(\mathrm{R}_{1}, \mathrm{~m}_{1}\right)$ and $\left(\mathrm{R}_{2}, \mathrm{~m}_{2}\right)$ are local rings, then $\left|\mathrm{Z}\left(\mathrm{R}_{1} \times \mathrm{R}_{2}\right)^{*}\right|=\left|\mathrm{R}_{1}\right| \mathrm{x}\left|\mathrm{m}_{2}\right|+\left|\mathrm{R}_{2}\right| \mathrm{x}\left|\mathrm{m}_{1}\right|-\left|\mathrm{m}_{1}\right|\left|\mathrm{m}_{2}\right|-1$.

In [5] we extended Wang's result. 
Lemma 2.2: If $\left(\mathrm{R}_{1}, \mathrm{~m}_{1}\right),\left(\mathrm{R}_{2}, \mathrm{~m}_{2}\right)$ and $\left(\mathrm{R}_{3}, \mathrm{~m}_{3}\right)$ are finite local rings, then $\left|\mathrm{Z}\left(\mathrm{R}_{1} \mathrm{xR}_{2} \mathrm{xR}_{3}\right)^{*}\right|=\left|\mathrm{R}_{1}\right| \mathrm{x}\left|\mathrm{R}_{2}\right| \mathrm{x}\left|\mathrm{m}_{3}\right|+\left|\mathrm{Z}\left(\mathrm{R}_{1} \times \mathrm{R}_{2}\right)\right| \mathrm{x}\left(\left|\mathrm{R}_{3}\right|-\left|\mathrm{m}_{3}\right|\right)-1$ where $\left|\mathrm{Z}\left(\mathrm{R}_{1} \mathrm{xR}_{2}\right)\right|=\left|\mathrm{R}_{1}\right| \mathrm{x}\left|\mathrm{m}_{2}\right|+\left|\mathrm{R}_{2}\right| \mathrm{x}\left|\mathrm{m}_{1}\right|-\left|\mathrm{m}_{1}\right| \mathrm{x}\left|\mathrm{m}_{2}\right|$.

As a direct consequence to Lemma 2.2, we obtain the following:

Corollary 2.3: If $R_{1}, R_{2}$ and $R_{3}$ are finite fields, then $\left|\mathrm{Z}\left(\mathrm{R}_{1} \times \mathrm{R}_{2} \times \mathrm{R}_{3}\right)^{*}\right|=\left|\mathrm{R}_{1}\right|\left|\mathrm{R}_{2}\right|+\left|\mathrm{R}_{1}\right|\left|\mathrm{R}_{3}\right|+\left|\mathrm{R}_{2}\right|\left|\mathrm{R}_{3}\right|-\left|\mathrm{R}_{1}\right|-\left|\mathrm{R}_{2}\right|-\left|\mathrm{R}_{3}\right|$.

Corollary 2.4 : If $R$ is a finite ring and $R \cong R_{1} \times R_{2} \times R_{3}$, then $\left|Z(R)^{*}\right| \geq 13$ for some local ring $\mathrm{R}_{\mathrm{i}}$ but not field.

Corollary 2.5: If $R_{i}$ is local not field for some $1 \leq i_{1}, i_{2} \leq 3$, then $\left|Z(R)^{*}\right| \geq 27$.

Lemma 2.6: [6] Let $R$ be a ring and $R \cong R_{1} \times R_{2} \times R_{3}$, where $R_{i}$ is local for $i=1,2,3$, then

1- If $\left|\mathrm{R}_{\mathrm{i}}\right| \geq 3$ for some $\mathrm{i}_{1}, \mathrm{i}_{2}$, then $\left|\mathrm{Z}(\mathrm{R})^{*}\right| \geq 13$.

2- If $\left|R_{i}\right| \geq 4$ for some $i$, then $\left|Z(R)^{*}\right| \geq 12$.

Lemma 2.7: [6] Let $R R_{1} \times R_{2} \times R_{3} \times R_{4}$, where $R_{i}$ is local for every $i$. Then $\left|\mathrm{Z}(\mathrm{R})^{*}\right| \geq 14$.

Next, we prove two fundamental lemmas

Lemma 2.8 : Let $R$ be a ring with $\left|Z(R)^{*}\right|=11$, then $R \cong R_{1} \times R_{2}$, where $R_{1}$ and $R_{2}$ are local rings.

Proof: Let $R \cong R_{1} \times R_{2} \ldots x R_{n}$, where each $R_{i}$ is a local ring. If $n \geq 4$ or $n=3$ with $R_{i}$ not field for some $i=1,2$ and 3, then we have a contradiction , by Lemma 2.7 and Corollary 2.4 respectively. It is clear that if $n=1$, then $|Z(R)|=12$ and hence, it also a contradiction so that we can investigate the case when $n=3$ and $R_{i}$ are fields for each $\mathrm{i}=1,2,3$. By Corollary 2.3 , $\left|\mathrm{Z}\left(\mathrm{R}_{1} \mathrm{xR}_{2} \times \mathrm{R}_{3}\right)^{*}\right|=\left|\mathrm{R}_{1}\right|\left|\mathrm{R}_{2}\right|+\left|\mathrm{R}_{1}\right|\left|\mathrm{R}_{3}\right|+\left|\mathrm{R}_{2}\right|\left|\mathrm{R}_{3}\right|-\left|\mathrm{R}_{1}\right|-\left|\mathrm{R}_{2}\right|-\left|\mathrm{R}_{3}\right|=11$. If $\left|R_{1}\right|=\left|R_{2}\right|=2$, then $\left|R_{3}\right|=11 / 3$ which is a contradiction. If $\left|R_{1}\right|=2,\left|R_{2}\right|=3$, then $\left|R_{3}\right|=5 / 2$, which is a contradiction. If $\left|R_{1}\right|=2$ and $\left|R_{2}\right| \geq 4$, then by Lemma $2.6(2)\left|Z(R)^{*}\right| \geq 12$, which is a contradiction. If $\left|R_{1}\right|$ and $\left|R_{2}\right| \geq 3$, then by Lemma2.6(1) $\left|Z(R)^{*}\right| \geq 13$, which is again a contradiction. Therefore, $n=2$ and, hence $R \cong R_{1} \times R_{2}$.

Lemma 2.9: Let $R$ be a ring with $\left|Z(R)^{*}\right|=11$. Then, $R \cong Z_{4} x Z_{4}, Z_{4} x Z_{2}[X] /\left(X^{2}\right)$, $\mathrm{Z}_{2}[\mathrm{X}] /\left(\mathrm{X}^{2}\right) \mathrm{x} \quad \mathrm{Z}_{2}[\mathrm{X}] /\left(\mathrm{X}^{2}\right), \quad \mathrm{Z}_{2} \mathrm{XZ}, \quad \mathrm{Z}_{2} \mathrm{XZ} \mathrm{Z}_{3}[\mathrm{X}] /\left(\mathrm{X}^{2}\right), \quad \mathrm{Z}_{2} \mathrm{XZ}, \quad \mathrm{Z}_{2} \mathrm{XZ} \mathrm{Z}_{2}[\mathrm{X}] /\left(\mathrm{X}^{3}\right)$, $\mathrm{Z}_{2} \mathrm{XZ} \mathrm{Z}_{4}[\mathrm{X}] /\left(2 \mathrm{X}, \mathrm{X}^{2}-2\right), \quad \mathrm{Z}_{2} \mathrm{XZ} \mathrm{Z}_{2}[\mathrm{X}, \mathrm{Y}] /(\mathrm{X}, \mathrm{Y})^{3}, \quad \mathrm{Z}_{2} \mathrm{XZ} \mathrm{Z}_{4}[\mathrm{X}] /\left(\mathrm{X}^{2}, 2 \mathrm{X}\right), \quad \mathrm{Z}_{5} \mathrm{XZ} \mathrm{Z}_{4}, \mathrm{Z}_{5} \mathrm{XZ} \mathrm{Z}_{2}[\mathrm{X}] /\left(\mathrm{X}^{2}\right)$, $\mathrm{Z}_{2} \mathrm{XZ}_{11}, \mathrm{~F}_{4} \mathrm{xF}_{9}$ or $\mathrm{Z}_{5} \mathrm{xF}$.

Proof: By Lemma 2.8; $\mathrm{R} \cong \mathrm{R}_{1} \times \mathrm{R}_{2}$, where $\mathrm{R}_{1}, \mathrm{R}_{2}$ are local rings. If $\mathrm{R}_{1}$ and $\mathrm{R}_{2}$ are not fields, then $\left|\mathrm{Z}\left(\mathrm{R}_{1} \times \mathrm{R}_{2}\right)^{*}\right|=\left|\mathrm{R}_{1}\right| \mathrm{x}\left|\mathrm{m}_{2}\right|+\left|\mathrm{R}_{2}\right| \mathrm{x}\left|\mathrm{m}_{1}\right|-\left|\mathrm{m}_{1}\right|\left|\mathrm{m}_{2}\right|-1=11$. If $\left|\mathrm{m}_{1}\right|=\mathrm{p}$, where $\mathrm{p}$ is prime, then $\left|R_{1}\right|=p^{2}$ [ 6, Lemma 4.8]. If $\left|m_{1}\right|=2$, then $\left|R_{1}\right|=4$ which implies that $\left|R_{2}\right|=6$ $\left|\mathrm{m}_{2}\right|$, therefore $\left|\mathrm{m}_{2}\right|=2$ and $\left|\mathrm{R}_{2}\right|=4$ so that $\mathrm{R} \cong \mathrm{Z}_{4} \mathrm{XZ} \mathrm{Z}_{4}$ or $\mathrm{Z}_{4} \mathrm{XZ} \mathrm{Z}_{2}[\mathrm{X}] /\left(\mathrm{X}^{2}\right)$ or $\mathrm{Z}_{2}[\mathrm{X}] /\left(\mathrm{X}^{2}\right) \mathrm{X}$ $\mathrm{Z}_{2}[\mathrm{X}] /\left(\mathrm{X}^{2}\right)$. if $\left|\mathrm{m}_{1}\right|=3$, then $\left|\mathrm{R}_{1}\right|=9$ which implies that $\left|\mathrm{R}_{2}\right|=4-2\left|\mathrm{~m}_{2}\right|$, but $\left|\mathrm{m}_{2}\right| \geq 2$, therefore $\left|R_{2}\right| \leq 0$ which is a contradiction. If $\left|\mathrm{m}_{1}\right|,\left|\mathrm{m}_{2}\right| \geq 4$, then $\left|\mathrm{R}_{1}\right|,\left|\mathrm{R}_{2}\right| \geq 8$ so that $11=\left|\mathrm{Z}(\mathrm{R})^{*}\right| \geq 47$ which is a contradiction. If $R_{1}$ is a field and $R_{2}$ is not a field, then $\left|R_{2}\right|=12-\left|m_{2}\right|\left(\left|R_{1}\right|-1\right)$. Let $\left|R_{1}\right|=2$, then $\left|R_{2}\right|=12-\left|m_{2}\right|$. Therefore, $\left|m_{2}\right|=3,\left|R_{2}\right|=9$ or $\left|m_{2}\right|=4,\left|R_{2}\right|=8$ and, hence $\mathrm{R} \cong \mathrm{Z}_{2} \mathrm{XZ}, \quad \mathrm{Z}_{2} \mathrm{XZ} \mathrm{Z}_{3}[\mathrm{X}] /\left(\mathrm{X}^{2}\right), \quad \mathrm{Z}_{2} \mathrm{XZ}, \quad \mathrm{Z}_{2} \mathrm{XZ} \mathrm{Z}_{2}[\mathrm{X}] /\left(\mathrm{X}^{3}\right) \quad \mathrm{Z}_{2} \mathrm{XZ} \mathrm{Z}_{4}[\mathrm{X}] /\left(2 \mathrm{X}, \mathrm{X}^{2}-2\right)$, $\mathrm{Z}_{2} \mathrm{XZ} \mathrm{Z}_{2}[\mathrm{X}, \mathrm{Y}] /(\mathrm{X}, \mathrm{Y})^{3}$ or $\left.\mathrm{Z}_{2} \mathrm{XZ} 4 \mathrm{X}\right] /\left(\mathrm{X}^{2}, 2 \mathrm{X}\right)$.

Let $\left|R_{1}\right|=3$, then $\left|R_{2}\right|=12-2\left|m_{2}\right|$, which is a contradiction. Let $\left|R_{1}\right|=4$ : Then, $\left|R_{2}\right|=12-3\left|m_{2}\right|$, which is also a contradiction. Let $\left|R_{1}\right|=5$. Then, $\left|R_{2}\right|=12-4\left|m_{2}\right|$. Therefore, $\left|\mathrm{m}_{2}\right|=2$ and $\left|\mathrm{R}_{2}\right|=4$ so that $\mathrm{R} \cong \mathrm{Z}_{5} \mathrm{XZ} \mathrm{Z}_{4}$ or $\mathrm{Z}_{5} \mathrm{x} \mathrm{Z}_{2}[\mathrm{X}] /\left(\mathrm{X}^{2}\right)$. Let $\left|\mathrm{R}_{1}\right| \geq 7$ : Then, $\left|\mathrm{R}_{2}\right|=12-6\left|\mathrm{~m}_{2}\right|$ and since $\left|m_{2}\right| \geq 2$, then $\left|R_{2}\right| \leq 0$ which is a contradiction. If $R_{1}$ and $R_{2}$ are fields, then applying Lemma $2.1\left|\mathrm{R}_{1}\right|+\left|\mathrm{R}_{2}\right|=13$ and hence $\left|\mathrm{R}_{1}\right|=2,\left|\mathrm{R}_{2}\right|=11$ or $\left|\mathrm{R}_{1}\right|=4,\left|\mathrm{R}_{2}\right|=9$ or $\left|\mathrm{R}_{1}\right|=5$, $\left|\mathrm{R}_{1}\right|=8$. Therefore, $\mathrm{R} \cong \mathrm{Z}_{2} \mathrm{xZ}_{11}, \mathrm{~F}_{4} \mathrm{xF}_{9}$ or $\mathrm{Z}_{5} \mathrm{xF}_{8}$.

Now, we shall prove the main result of this section. 
Theorem 2.10: Let $\mathrm{R}$ be a ring with $|\mathrm{Z}(\mathrm{R}) *|=11$, then, the graphs depicted in the following figures can be realized as $\Gamma(\mathrm{R})$.

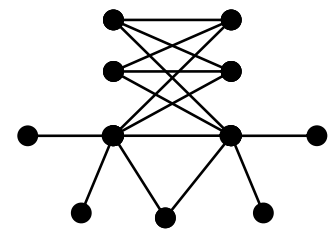

Figure (1)

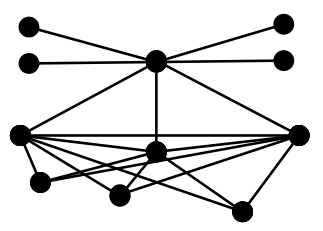

Figure ( 5)

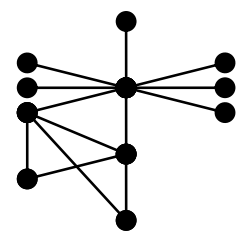

Figure (2)

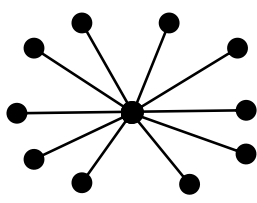

Figure ( 6)

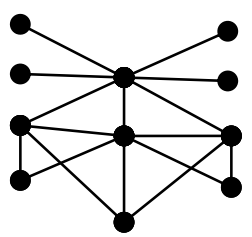

Figure (3)

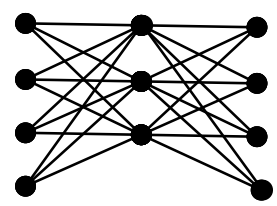

Figure ( 7 )

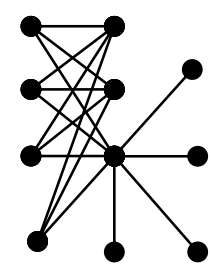

Figure (4)

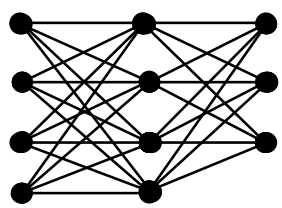

Figure ( 8 )

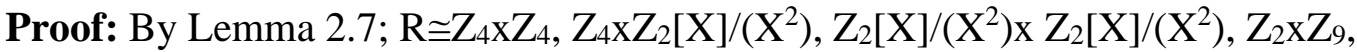
$\mathrm{Z}_{2} \mathrm{XZ} \mathrm{Z}_{3}[\mathrm{X}] /\left(\mathrm{X}^{2}\right), \mathrm{Z}_{2} \mathrm{XZ}, \mathrm{Z}_{2} \mathrm{XZ} \mathrm{Z}_{2}[\mathrm{X}] /\left(\mathrm{X}^{3}\right), \mathrm{Z}_{2} \mathrm{XZ}$ [ $[\mathrm{X}] /\left(2 \mathrm{X}, \mathrm{X}^{2}-2\right), \mathrm{Z}_{2} \mathrm{xZ} \mathrm{Z}_{2}[\mathrm{X}, \mathrm{Y}] /(\mathrm{X}, \mathrm{Y})^{3}$, $\mathrm{Z}_{2} \mathrm{XZ} \mathrm{Z}_{4}[\mathrm{X}] /\left(\mathrm{X}^{2}, 2 \mathrm{X}\right), \mathrm{Z}_{5} \mathrm{xZ} \mathrm{Z}_{4}, \mathrm{Z}_{5} \mathrm{xZ} \mathrm{Z}_{2}[\mathrm{X}] /\left(\mathrm{X}^{2}\right), \mathrm{Z}_{2} \mathrm{XZ} \mathrm{Z}_{11}, \mathrm{~F}_{4} \mathrm{XF} \mathrm{F}_{9}$ or $\mathrm{Z}_{5} \mathrm{xF}$. Figure (1), can be realized as $\Gamma\left(\mathrm{Z}_{4} \mathrm{xZ} \mathrm{Z}_{4}\right)$ or $\Gamma\left(\mathrm{Z}_{4} \mathrm{XZ} \mathrm{Z}_{2}[\mathrm{X}] /\left(\mathrm{X}^{2}\right)\right)$ or $\Gamma\left(\mathrm{Z}_{2}[\mathrm{X}] /\left(\mathrm{X}^{2}\right) \mathrm{x} \mathrm{Z}_{2}[\mathrm{X}] /\left(\mathrm{X}^{2}\right)\right)$. Figure (2), can be realized as $\Gamma\left(Z_{2} x Z_{9}\right)$ or $\Gamma\left(Z_{2} X Z_{3}[X] /\left(X^{2}\right)\right)$. Figure (3), can be realized as $\Gamma\left(Z_{2} x Z_{8}\right)$ or $\Gamma\left(Z_{2} Z_{2}[X] /\left(X^{3}\right)\right)$ or $Z_{2} X Z_{4}[X] /\left(2 X, X^{2}-2\right)$. Figure (4), can be realized as $\Gamma\left(Z_{5} x Z_{4}\right)$ or $\Gamma\left(Z_{5} X_{2}[X] /\left(X^{2}\right)\right)$. Figure $(5)$, can be realized $\Gamma\left(Z_{2} X Z_{4}[X] /\left(2 X, X^{2}\right)\right)$ or $\Gamma\left(\mathrm{Z}_{2} \mathrm{XZ}[\mathrm{X}, \mathrm{Y}] /(\mathrm{X}, \mathrm{Y})^{2}\right)$. Figure (6), can be realized as $\Gamma\left(\mathrm{Z}_{2} \mathrm{XZ} \mathrm{Z}_{11}\right)$. Figure (7), can be realized as $\Gamma\left(\mathrm{F}_{4} \mathrm{XF}_{9}\right)$ and Figure $(8)$, can be realized as $\Gamma\left(\mathrm{Z}_{5} \mathrm{XF}_{8}\right)$.

\section{Rings with $|\mathrm{Z}(\mathrm{R}) *|=12$}

The main aim of this section is to find all possible zero divisor graphs of 12 vertices and rings correspond to them.

We shall start this section with the following lemmas.

Lemma 3.1 : Let $R$ be a ring with $\left|Z(R)^{*}\right|=12$; if $R \cong R_{1} \times R_{2} x \ldots R_{n}$, where $R_{i}$ is a local ring for all $i \geq 1$, then $n=3$ if and only if $R \cong Z_{2} \times Z_{2} \times F_{4}$

Proof: Let $R$ be a ring with $\left|Z(R)^{*}\right|=12$ and let $R \cong R_{1} \times R_{2} \times R_{3}$ where $R_{i}$ is a local ring for all $i=1,2,3$. If $R_{i}$ is not a field for some $1 \leq i \leq 3$, then $\left|Z(R)^{*}\right| \geq 13$ which is a contradiction, so that $R_{i}$ is a field for all $1 \leq i \leq 3$, then by Corollary2.3, $\left|Z\left(R_{1} \times R_{2} \times R_{3}\right)^{*}\right|=$ $\left|\mathrm{R}_{1}\right|\left|\mathrm{R}_{2}\right|+\left|\mathrm{R}_{1}\right|\left|\mathrm{R}_{3}\right|+\left|\mathrm{R}_{2}\right|\left|\mathrm{R}_{3}\right|-\left|\mathrm{R}_{1}\right|-\left|\mathrm{R}_{2}\right|-\left|\mathrm{R}_{3}\right|=12$. If $\left|\mathrm{R}_{1}\right|=\left|\mathrm{R}_{2}\right|=2$, then $\left|\mathrm{R}_{3}\right|=4$, so that $\mathrm{R} \cong \mathrm{Z}_{2} \times Z_{2} \times F_{4}$. If $\left|\mathrm{R}_{1}\right|=2$ and $\left|\mathrm{R}_{2}\right|=3$, then $\left|\mathrm{R}_{3}\right|=13 / 4$ which is a contradiction. If $\left|\mathrm{R}_{1}\right| \geq 3$ and $\left|R_{2}\right| \geq 3$, then by Lemma2.6(1) $\left|\mathrm{Z}(\mathrm{R})^{*}\right| \geq 13$, which is a contradiction.

Lemma 3.2: Let $R$ be a ring with $\left|Z(R)^{*}\right|=12$, if $R \cong R_{1} \times R_{2} x \ldots R_{n}$, where $R_{i}$ is a local ring for all $\mathrm{i} \geq 1$, then $\mathrm{n}=2$ if and only if $\mathrm{R} \cong \mathrm{Z}_{3} \times \mathrm{Z}_{11}, \mathrm{Z}_{5} \times F_{9}$ or $\mathrm{Z}_{7} \times \mathrm{Z}_{7}$

Proof: Let $R$ be a ring with $\left|Z(R)^{*}\right|=12$ and let $R \cong R_{1} x R_{2}$ where $R_{1}$ and $R_{2}$ are local rings. If $\mathrm{R}_{1}$ and $\mathrm{R}_{2}$ are not fields, then $\left|\mathrm{Z}\left(\mathrm{R}_{1} \mathrm{xR}_{2}\right)^{*}\right|=\left|\mathrm{R}_{1}\right| \mathrm{x}\left|\mathrm{m}_{2}\right|+\left|\mathrm{R}_{2}\right| \mathrm{x}\left|\mathrm{m}_{1}\right|-\left|\mathrm{m}_{1}\right|\left|\mathrm{m}_{2}\right|-1=12$.

If $\left|\mathrm{m}_{1}\right|=2$, then $\left|\mathrm{R}_{1}\right|=4$. So that, $\left|\mathrm{R}_{2}\right|=13 / 2-\left|\mathrm{m}_{2}\right|$. Since, $\left|\mathrm{m}_{2}\right|$ is an integer, then $\left|\mathrm{R}_{2}\right|$ is not an integer which is a contradiction.

If $\left|\mathrm{m}_{1}\right| \geq 3$, then $\left|\mathrm{R}_{1}\right| \geq 8$ and, since $\left|\mathrm{m}_{2}\right| \geq 2,\left|\mathrm{R}_{2}\right| \geq 4$, then $12=\left|\mathrm{Z}(\mathrm{R})^{*}\right| \geq 21$ which is also a contradiction.

If $\left|R_{1}\right|$ is a field and $\left|R_{2}\right|$ is not a field, then $\left|R_{2}\right|+\left|m_{2}\right|\left|R_{1}\right|-\left|m_{2}\right|=12$ which this leads to a contradiction. If $R_{1}$ and $R_{2}$ are fields, then $\left|R_{1}\right|+\left|R_{2}\right|=14$ which implies that $\left|R_{1}\right|=3,\left|R_{2}\right|=11$ or $\left|R_{1}\right|=5,\left|R_{2}\right|=9$ or $\left|R_{1}\right|=\left|R_{2}\right|=7$. Therefore $R \cong Z_{3} \times Z_{11}, Z_{5} \times F_{9}$ or $Z_{7} \times Z_{7}$. 
Lemma 3.3: Let $\mathrm{R}$ be a ring with $\left|\mathrm{Z}(\mathrm{R})^{*}\right|=12$. Then, $\mathrm{R} \cong \mathrm{Z}_{2} \mathrm{xZ}_{2} \mathrm{xF}_{4}, \mathrm{Z}_{3} \times \mathrm{Z}_{11}, \mathrm{Z}_{5} \times F_{9}$, $\mathrm{Z}_{7} \mathrm{XZ} \mathrm{Z}_{7}, \mathrm{Z}_{169}$ or $\mathrm{Z}_{13}[\mathrm{X}] /\left(\mathrm{X}^{2}\right)$

Proof: Let $R \cong R_{1} \times R_{2} \ldots x R_{n}$, where $R_{i}$ is a local ring. If $n \geq 4$, then by Lemma 2.7 $\left|\mathrm{Z}(\mathrm{R})^{*}\right| \geq 14$. If $\mathrm{n}=3$, then $\mathrm{R} \cong \mathrm{Z}_{2} \times \mathrm{Z}_{2} \times F_{4}$. If $\mathrm{n}=2$, then $\mathrm{R} \cong \mathrm{Z}_{3} \times \mathrm{Z}_{11} \mathrm{Z}_{5} \times F_{9}$ or $\mathrm{Z}_{7} \times \mathrm{Z}_{7}$. If $\mathrm{n}=1$ and $R$ is a field, then $Z(R)=\{0\}$ which is a contradiction. If $R$ is a local ring, then $\left|\mathrm{Z}(\mathrm{R})^{*}\right|=|\mathrm{m}|-1=12$, so that $|\mathrm{m}|=13$. Therefore, $|\mathrm{R}|=169$, which implies that $\mathrm{R} \cong \mathrm{Z}_{169}$ or $\mathrm{Z}_{13}[\mathrm{X}] /\left(\mathrm{X}^{2}\right)$.

Theorem 3.4: Let $\mathrm{R}$ be a ring with $|\mathrm{Z}(\mathrm{R})|^{*}=12$, then the graphs depicted in the following figures can be realized as $\Gamma(\mathrm{R})$

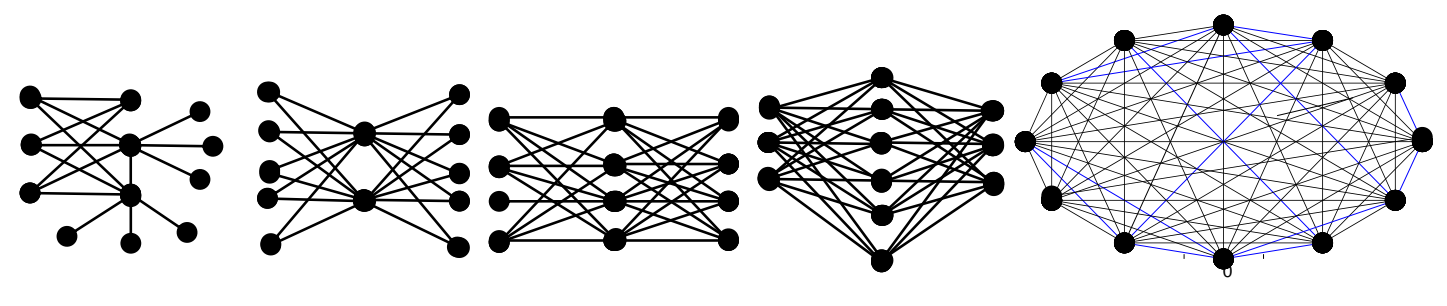

$\begin{array}{lllll}\text { Figure (1) } & \text { Figure (2) } & \text { Figure (3) } & \text { Figure (4) } & \text { Figure (5) }\end{array}$

Proof: By Lemma 3.3; $\mathrm{R} \cong \mathrm{Z}_{2} \mathrm{xZ}_{2} \mathrm{xF}_{4}, \mathrm{Z}_{3} \mathrm{XZ}_{11}, \mathrm{Z}_{5} \mathrm{xF} \mathrm{F}_{9}, \mathrm{Z}_{7} \mathrm{xZ} \mathrm{Z}_{7}, \mathrm{Z}_{169}$ or $\mathrm{Z}_{13}[\mathrm{X}] /\left(\mathrm{X}^{2}\right)$

. In Figure (1), can be realized as $\Gamma\left(Z_{2} \times Z_{2} \times F_{4}\right)$. Figure (2), can be realized as $\Gamma\left(Z_{3} \times Z_{11}\right)$. Figure (3), can be realized as $\Gamma\left(Z_{7} \times Z_{7}\right)$. Figure (4), can be realized as $\Gamma\left(Z_{7} \mathrm{X}_{7}\right)$. Figure $(5)$, can be realized as $\Gamma\left(\mathrm{Z}_{169}\right)$ or $\Gamma\left(\mathrm{Z}_{13}[\mathrm{X}] /(\mathrm{X})^{2}\right)$.

\section{Rings with $|\mathrm{Z}(\mathrm{R}) *|=13$}

The main aim of this section is to find all possible zero divisor graphs of 13 vertices and rings correspond to them.

We shall start this section with following lemma.

Lemma 4.1 : Let $R$ be a ring with $\left|Z(R)^{*}\right|=13$, if $R \cong R_{1} \times R_{2} x \ldots R_{n}$, where $R_{i}$ is a local ring for all $\mathrm{i} \geq 1$, then $\mathrm{n}=3$ if and only if $\mathrm{R} \cong \mathrm{Z}_{2} \times Z_{3} \times Z_{3}, Z_{2} \times Z_{2} \times Z_{4}$ or $\mathrm{Z}_{2} \mathrm{XZ}_{2} \mathrm{XZ} \mathrm{Z}_{2}[\mathrm{X}] /\left(\mathrm{X}^{2}\right)$.

Proof : Let $R$ be a ring with $\left|Z(R)^{*}\right|=13$ and let $R \cong R_{1} \times R_{2} \times R_{3}$, where $R_{i}$ local rings for all $1 \leq \mathrm{i} \leq 3$. If $\mathrm{R}_{\mathrm{i}}$ is not a field, for some $1 \leq \mathrm{i}_{1}, \mathrm{i}_{2} \leq 3$, then $\left|Z\left(\mathrm{R}_{1} \times \mathrm{R}_{2} \times \mathrm{R}_{3}\right)^{*}\right| \geq 27$ which is a contradiction.

If $R_{3}$ is not a field and $R_{1}$ and $R_{2}$ are fields, then $\left|Z\left(R_{1} \times R_{2}\right)\right|=\left|R_{1}\right|+\left|R_{2}\right|-1$ and $\left|\mathrm{Z}\left(\mathrm{R}_{1} \mathrm{xR}_{2} \mathrm{x} \mathrm{R}_{3}\right)^{*}\right|=\left|\mathrm{R}_{1}\right|\left|\mathrm{R}_{2}\right|\left|\mathrm{m}_{3}\right|+\left(\left|\mathrm{R}_{1}\right|+\left|\mathrm{R}_{2}\right|-1\right)\left(\left|\mathrm{R}_{3}\right|-\left|\mathrm{m}_{3}\right|\right)-1$, so that $\left|\mathrm{R}_{1}\right|\left|\mathrm{R}_{2}\right|\left|\mathrm{m}_{3}\right|+\left(\left|\mathrm{R}_{1}\right|+\left|\mathrm{R}_{2}\right|-\right.$ $1)\left(\left|R_{3}\right|-\left|m_{3}\right|\right)=14$

If $\left|R_{1}\right|=\left|R_{2}\right|=2$, then $\left|R_{3}\right|=\frac{14-\left|m_{3}\right|}{3}$ which implies that $\left|R_{3}\right|=4$ and $\left|m_{3}\right|=2$. Therefore,

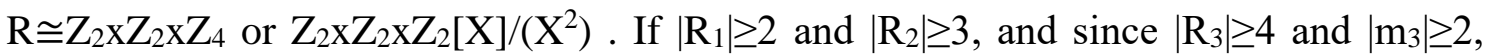
then $13=\left|Z\left(R_{1} \times R_{2} \times R_{3}\right)^{*}\right| \geq 2.3 .2+(2+3-1)(4-2)-1 \geq 19$ which is a contradiction. If $R_{i}$ is a field for all $1 \leq \mathrm{i} \leq 3$, then

$\left|R_{1}\right|\left|R_{2}\right|+\left|R_{1}\right|\left|R_{3}\right|+\left|R_{2}\right|\left|R_{3}\right|-\left|R_{1}\right|-\left|R_{2}\right|-\left|R_{3}\right|=13$. If $\left|R_{1}\right|=\left|R_{2}\right|$, then $\left|R_{3}\right|=13 / 2$ which is a contradiction. If $\left|R_{1}\right|=2,\left|R_{2}\right|=3$, then $\left|R_{3}\right|=3$ so that $R \cong Z_{2} x Z_{3} x Z_{3}$. If $\left|R_{1}\right|=2$ and $\left|R_{2}\right|=4$, then $\left|R_{3}\right|=11 / 5$ which is a contradiction. If $\left|R_{1}\right|=2$ and $\left|R_{2}\right|=5$, then $\left|R_{3}\right|=5 / 3$ which is a contradiction. If $\left|R_{1}\right|=2$ and $\left|R_{2}\right| \geq 7$, then $\left|R_{3}\right| \leq 1$ which is a contradiction. If $\left|R_{1}\right| \geq 3$ and $\left|R_{2}\right| \geq 4$, then $\left|R_{3}\right| \leq 4 / 3$ which is a contradiction.

Lemma 4.2 : Let $R$ be a ring with $\left|Z(R)^{*}\right|=13$, if $R \cong R_{1} \times R_{2} x \ldots R_{n}$, where $R_{i}$ is a local ring for all $\mathrm{i} \geq 1$, then $\mathrm{n}=2$ if and only if $\mathrm{R} \cong \mathrm{Z}_{2} \times \mathrm{Z}_{13}, \mathrm{~F}_{4} \times \mathrm{Z}_{11}$ or $\mathrm{Z}_{7} \times \mathrm{F}_{8}$. 
Proof : Let $\mathrm{R}$ be a ring with $\left|\mathrm{Z}(\mathrm{R})^{*}\right|=13$ and let $\mathrm{R} \cong \mathrm{R}_{1} \times \mathrm{R}_{2}$, where $\mathrm{R}_{1}$ and $\mathrm{R}_{2}$ local rings. If $R_{1}$ and $R_{2}$ are not fields, then

$\left|\mathrm{Z}\left(\mathrm{R}_{1} \times \mathrm{R}_{2}\right)^{*}\right|=\left|\mathrm{R}_{1}\right|\left|\mathrm{m}_{2}\right|+\left|\mathrm{R}_{2}\right|\left|\mathrm{m}_{1}\right|-\left|\mathrm{m}_{1}\right|\left|\mathrm{m}_{2}\right|=14$. If $\left|\mathrm{m}_{1}\right|=2$, then $\left|\mathrm{R}_{1}\right|=4$, so that $\left|\mathrm{R}_{2}\right|=7-\left|\mathrm{m}_{2}\right|$ which is a contradiction. If $\left|\mathrm{m}_{1}\right|,\left|\mathrm{m}_{2}\right| \geq 3$, then $\left|\mathrm{R}_{1}\right|,\left|\mathrm{R}_{2}\right| \geq 8$, so that $\left|\mathrm{Z}\left(\mathrm{R}_{1} \times \mathrm{R}_{2}\right)^{*}\right| \geq 3$.8+8.3-3.3$1=38$ which is a contradiction. If $R_{1}$ field and $R_{2}$ local not field, then $\left|R_{1}\right|\left|m_{2}\right|+\left|R_{2}\right|-$ $\left|\mathrm{m}_{2}\right|=14$ which implies that $\left|\mathrm{R}_{2}\right|=14-\left(\left|\mathrm{R}_{1}\right|-1\right)\left|\mathrm{m}_{2}\right|$. If $\left|\mathrm{R}_{1}\right|=2$, then $\left|\mathrm{R}_{2}\right|=14-\left|\mathrm{m}_{2}\right|$ which is a contradiction. If $\left|R_{1}\right|=3$, then $\left|R_{2}\right|=14-2\left|m_{2}\right|$ which is a contradiction. If $\left|R_{1}\right|=4$, then $\left|\mathrm{R}_{2}\right|=14-3\left|\mathrm{~m}_{2}\right|$ which is a contradiction. If $\left|\mathrm{R}_{1}\right|=5$, then $\left|\mathrm{R}_{2}\right|=14-4\left|\mathrm{~m}_{2}\right|$ which is a contradiction. If $\left|R_{1}\right| \geq 7$, then $\left|Z\left(R_{1} \times R_{2}\right)^{*}\right| \geq 15$ which is a contradiction. Therefore, $R_{1}$ and $\mathrm{R}_{2}$ are fields, which imply that $\left|\mathrm{R}_{1}\right|+\left|\mathrm{R}_{2}\right|=15$ and, hence $\left|\mathrm{R}_{1}\right|=2,\left|\mathrm{R}_{2}\right|=13$ or $\left|\mathrm{R}_{1}\right|=4,\left|\mathrm{R}_{2}\right|=11$ or $\left|\mathrm{R}_{1}\right|=7,\left|\mathrm{R}_{2}\right|=8$. Therefore, $\mathrm{R} \cong \mathrm{Z}_{2} \mathrm{xZ}_{13}, \mathrm{~F}_{4} \times \mathrm{Z}_{11}$ or $\mathrm{Z}_{7} \times \mathrm{F}_{8}$.

Lemma 4.3: Let $R$ be a ring with $\left|Z(R)^{*}\right|=13$, then $R \cong Z_{2} x Z_{2} x Z_{4}$, $\mathrm{Z}_{2} \mathrm{XZ}_{2} \mathrm{xZ} \mathrm{Z}_{2}[\mathrm{X}] /\left(\mathrm{X}^{2}\right), \mathrm{R} \cong \mathrm{Z}_{2} \mathrm{xZ} \mathrm{Z}_{13}, \mathrm{~F}_{4} \mathrm{xZ} \mathrm{Z}_{11}$ or $\mathrm{Z}_{7} \mathrm{XF}_{8}$.

Proof: Let $R \cong R_{1} \times R_{2} \ldots x R_{n}$, where $R_{i}$ is a local ring. If $n \geq 4$, then by Lemma 2.7 $\left|Z(R)^{*}\right| \geq 14$. If $n=3$, then $R \cong Z_{2} x Z_{2} x Z_{4}$ or $Z_{2} x Z_{2} x Z_{2}[X] /\left(X^{2}\right)$ Lemma 4.1. If $n=2$, then $\mathrm{R} \cong \mathrm{Z}_{2} \times Z_{13}, \mathrm{~F}_{4} \times Z_{11}$ or $\mathrm{Z}_{7} \times F_{8}$ Lemma4.2. If $\mathrm{n}=1$ and $\mathrm{R}$ is a field, then $\mathrm{Z}(\mathrm{R})=\{0\}$ which is a contradiction. If $R$ is a local ring, then $\left|Z(R)^{*}\right|=m-1=13$, so that $|m|=14$ which is also a contradiction.

Theorem 4.4: Let $\mathrm{R}$ be a ring with $|\mathrm{Z}(\mathrm{R})|^{*}=13$, then the graphs depicted in the following figures can be realized as $\Gamma(\mathrm{R})$

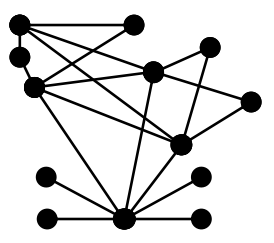

Figure (1)

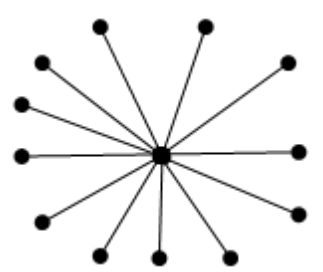

Figure (2)

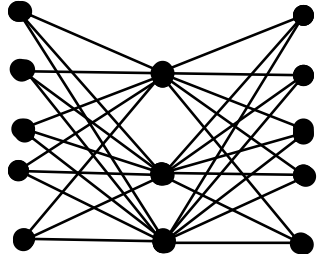

Figure (3)

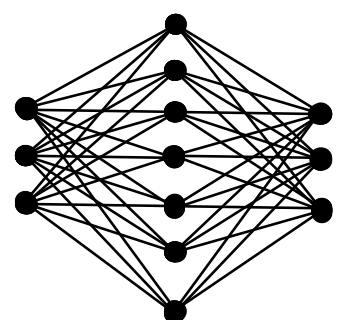

Figure (4)

Proof: By Lemma 4.3 $\mathrm{R} \cong \mathrm{Z}_{2} \mathrm{XZ}_{2} \mathrm{xZ} \mathrm{Z}_{4}, \mathrm{Z}_{2} \mathrm{xZ} \mathrm{Z}_{2} \mathrm{xZ}[\mathrm{X}] /\left(\mathrm{X}^{2}\right), \mathrm{Z}_{2} \mathrm{xZ} \mathrm{Z}_{13}, \mathrm{~F}_{4} \mathrm{xZ} \mathrm{Z}_{11}$ or $\mathrm{Z}_{7} \mathrm{xF}$. Figure (1) can be realized as $\Gamma\left(Z_{2} x Z_{2} x Z_{4}\right)$ or $\Gamma\left(Z_{2} x Z_{2} x Z_{2}[X] /(X)^{2}\right.$. Figure (2) can be realized as $\Gamma\left(Z_{2} x Z_{13}\right)$. Figure (3) can be realized as $\Gamma\left(F_{4} x Z_{11}\right)$ and Figure (4) can be realized as $\Gamma\left(\mathrm{Z}_{7} \mathrm{x} \mathrm{Z}_{8}\right)$.

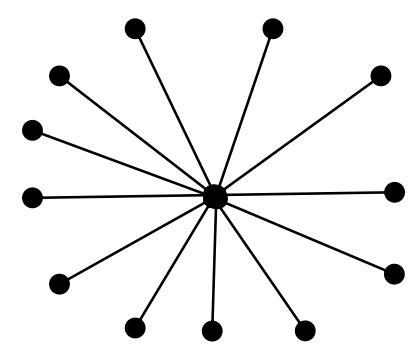




\section{REFERENCES}

[1] D.F. Andersen and P. S. Livingston ,(1999), "The Zero Divisor Graph of a Commutative Ring". Journal of Algebra 217, pp. 434-447.

[2] A. Badawi, (2004), "Abstract Algebra Manual: Problems and Solutions 2nd Edition problems and solutions", Nova Science Publishe.

[3] I. Beck , (1988), "Coloring of Commutative Ring". Journal of Algebra 116, pp. 208-226.

[4] G. Carbas and D. Williams, (2000), " Rings of Order p ${ }^{5}$.I Nonlocal Rings " , Journal of Algebra 231(2), pp. 677-690.

[5] N.H. Shuker and H. Q. Mohammad , (2012), "Classification of Zero Divisor Graphs of a Commutative Ring with Degree Equal 7 and 8" , Accepted on AL-Rafidain Journal of Computer Science and Mathematics.

[6] J. T. Wang, (2005), "Zero Divisor of Commutative Rings", M.Sc. Thesis at the University of National Chung Cheng, Taiwan. 